\title{
1 Air quality in Delhi during the Commonwealth Games
}

2 Pallavi Marrapu ${ }^{\mathrm{a}, \mathrm{b}, \mathrm{e}}$, YafangCheng ${ }^{\mathrm{b}, \mathrm{c}}$, GufranBeig $^{\mathrm{d}}$, S. Sahu ${ }^{\mathrm{d}}$, R.Srinivas ${ }^{\mathrm{d}}$ and Gregory

3 R.Carmichael ${ }^{\mathrm{a}, \mathrm{b}}$

4 a) Department of Chemical and Biochemical Engineering, University of Iowa, Iowa city, USA 52242.

b) Center for Global and Regional Environmental Research, University of Iowa, Iowa city, USA 52242.

c) Multiphase Chemistry Department, Max Planck Institute Chemistry, Hahn-Meitner-Weg1, Mainz, Germany 55128.

d) Indian Institute of Tropical Meteorology (Ministry of Earth Sciences, Govt. of India) Dr. HomiBhabha Road, Pashan, Pune, India 411008.

e) Molina Center for Strategic Studies in Energy and Environment, La Jolla, California, USA 92037.

Supplemental Information 
45 Table S1 Comparison of 20-day mean observed and predicted concentrations of various species

46 at all monitoring sites with Original Emissions

47

\begin{tabular}{|c|c|c|c|c|c|c|c|}
\hline $\begin{array}{c}\mathrm{NO}_{\mathrm{x}^{-}} \\
\text {Full }\end{array}$ & $\begin{array}{c}\mathrm{BC} \\
\left(\mu \mathrm{gg} / \mathrm{m}^{3}\right)\end{array}$ & $\begin{array}{c}\mathrm{CO} \\
(\mathrm{ppmv})\end{array}$ & $\begin{array}{c}\mathrm{NO}_{\mathrm{X}} \\
(\mathrm{ppbv})\end{array}$ & $\begin{array}{c}\mathrm{O}_{3} \\
(\mathrm{ppbv})\end{array}$ & $\begin{array}{c}\mathbf{O}_{3^{-}} \\
\text {DayTime(ppbv) }\end{array}$ & $\begin{array}{c}\mathrm{PM}_{2.5} \\
(\mu \mathrm{g} / \mathrm{m3})\end{array}$ & $\begin{array}{c}\mathrm{PM}_{10} \\
(\mu \mathrm{gg} / \mathrm{m3})\end{array}$ \\
\hline $\begin{array}{c}\text { Mean- } \\
\text { obs }\end{array}$ & 9.5 & 1.7 & 67.8 & 30.1 & 54.5 & 110.8 & 237.6 \\
\hline $\begin{array}{c}\text { Mean- } \\
\text { model }\end{array}$ & 13.8 & 1.0 & 150.3 & 26.3 & 60.4 & 181.5 & 294.3 \\
\hline Bias & 10.1 & -0.5 & 91.3 & 0.3 & 12.5 & 83.6 & 83.5 \\
\hline RMSE & 15.7 & 1.4 & 170.3 & 29.2 & 40.6 & 162.8 & 259.5 \\
\hline R & 0.6 & 0.4 & 0.4 & 0.7 & 0.4 & 0.4 & 0.4 \\
\hline
\end{tabular}




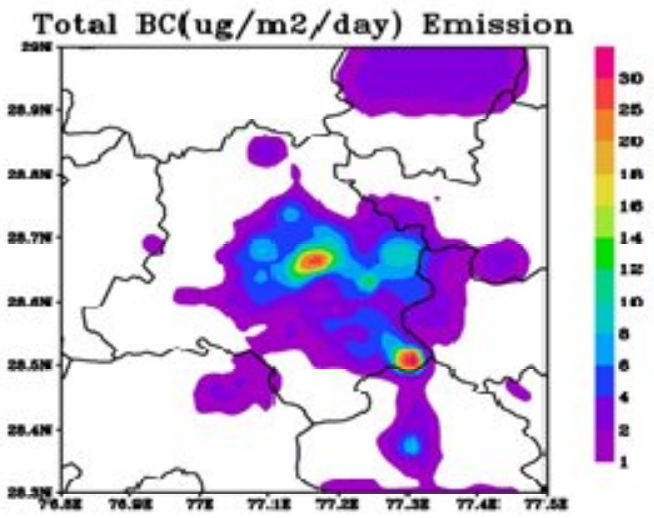

Total NOx(kmol/km2/day) Emission

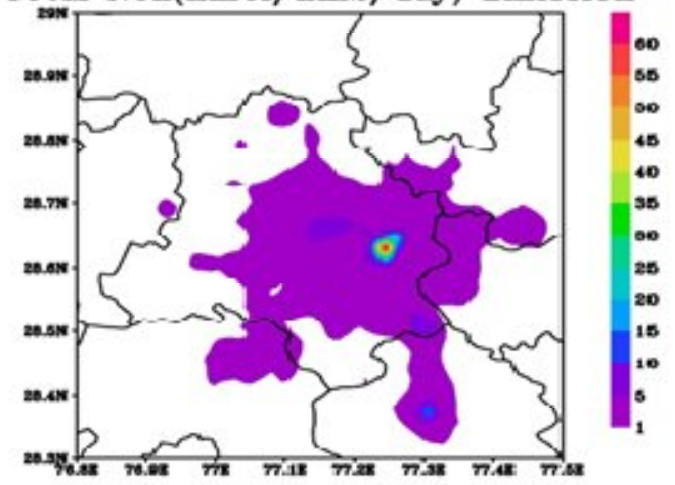

Total suz(kmol/kmz/day) Kmission

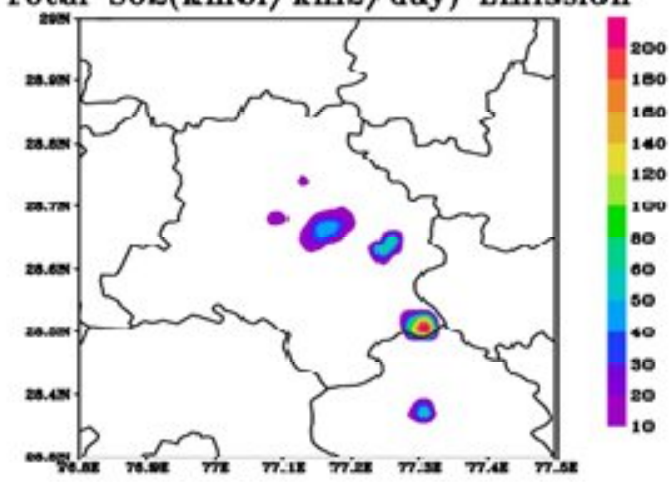

Total $\mathrm{CO}(\mathrm{kmol} / \mathrm{km} /$ day $)$ Emission

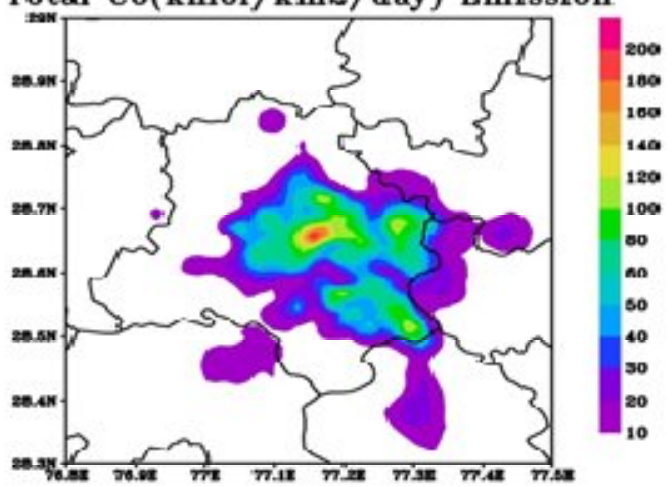

Total PM2_5(ug/m2/day) Emission

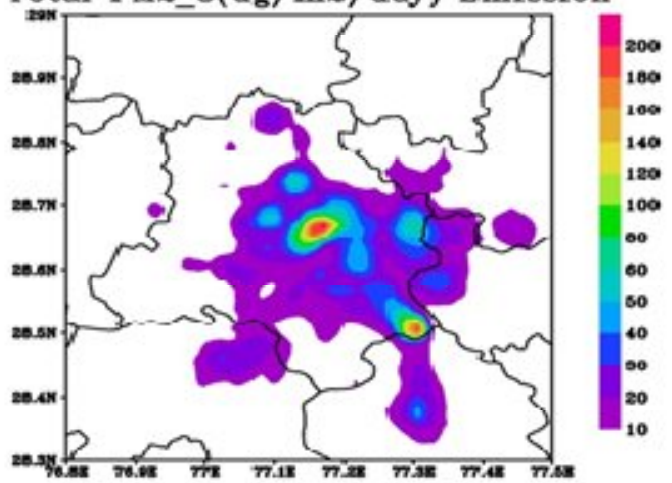

Fig S1 .Emission distributions of $\mathrm{BC}, \mathrm{CO}, \mathrm{NO}_{\mathrm{x}}, \mathrm{PM}_{2.5}$ and $\mathrm{SO}_{2}$ units over Delhi. 

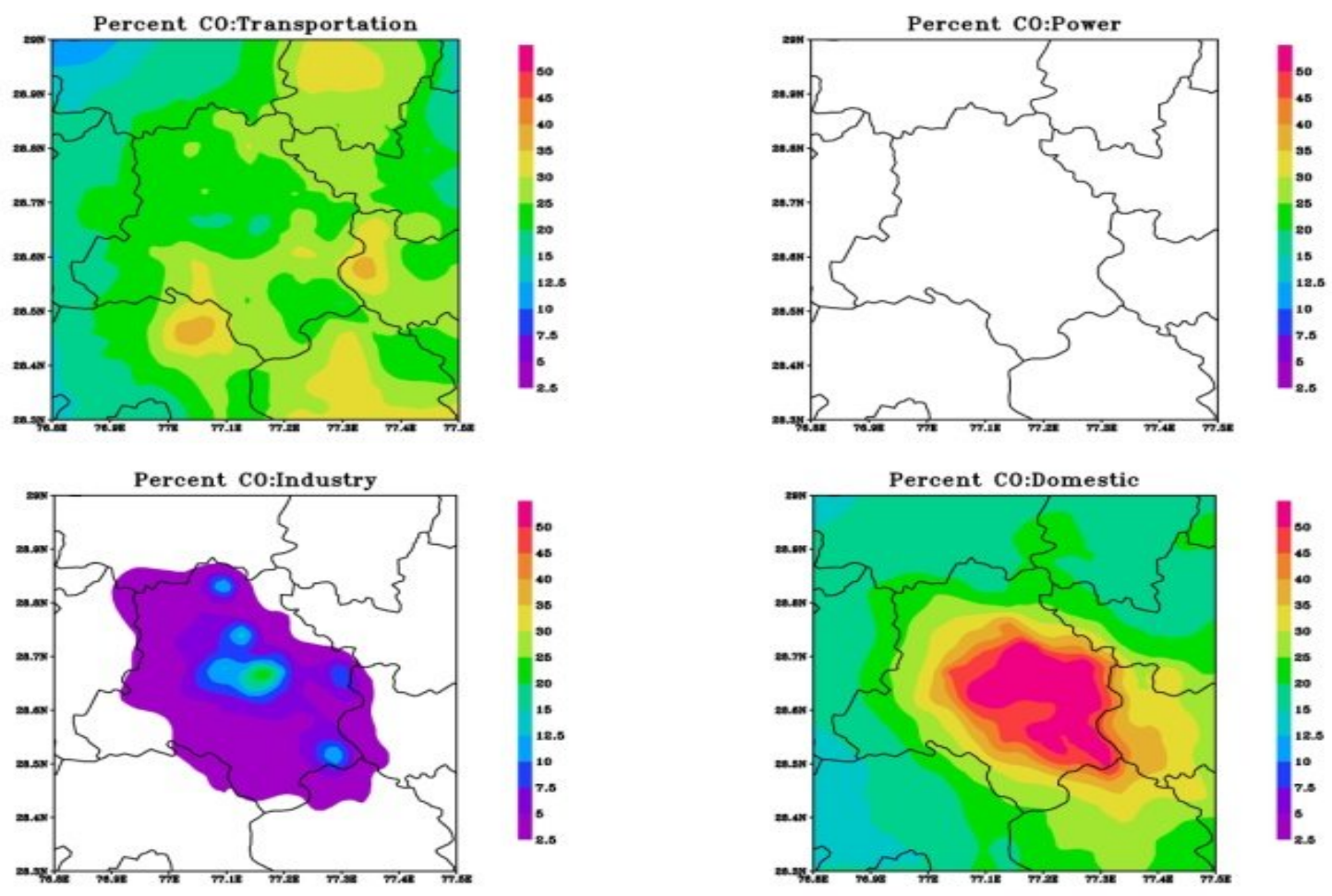

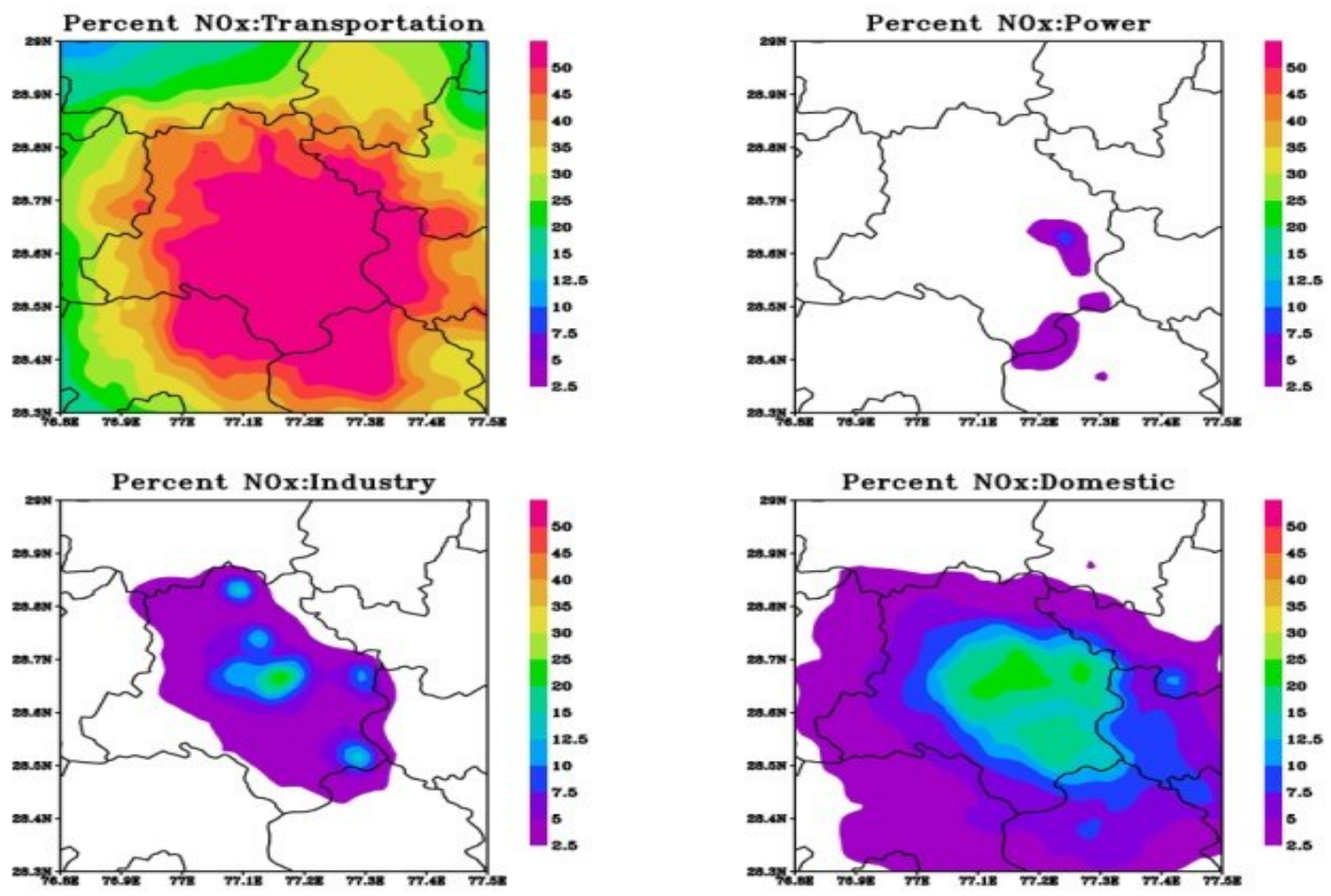

52
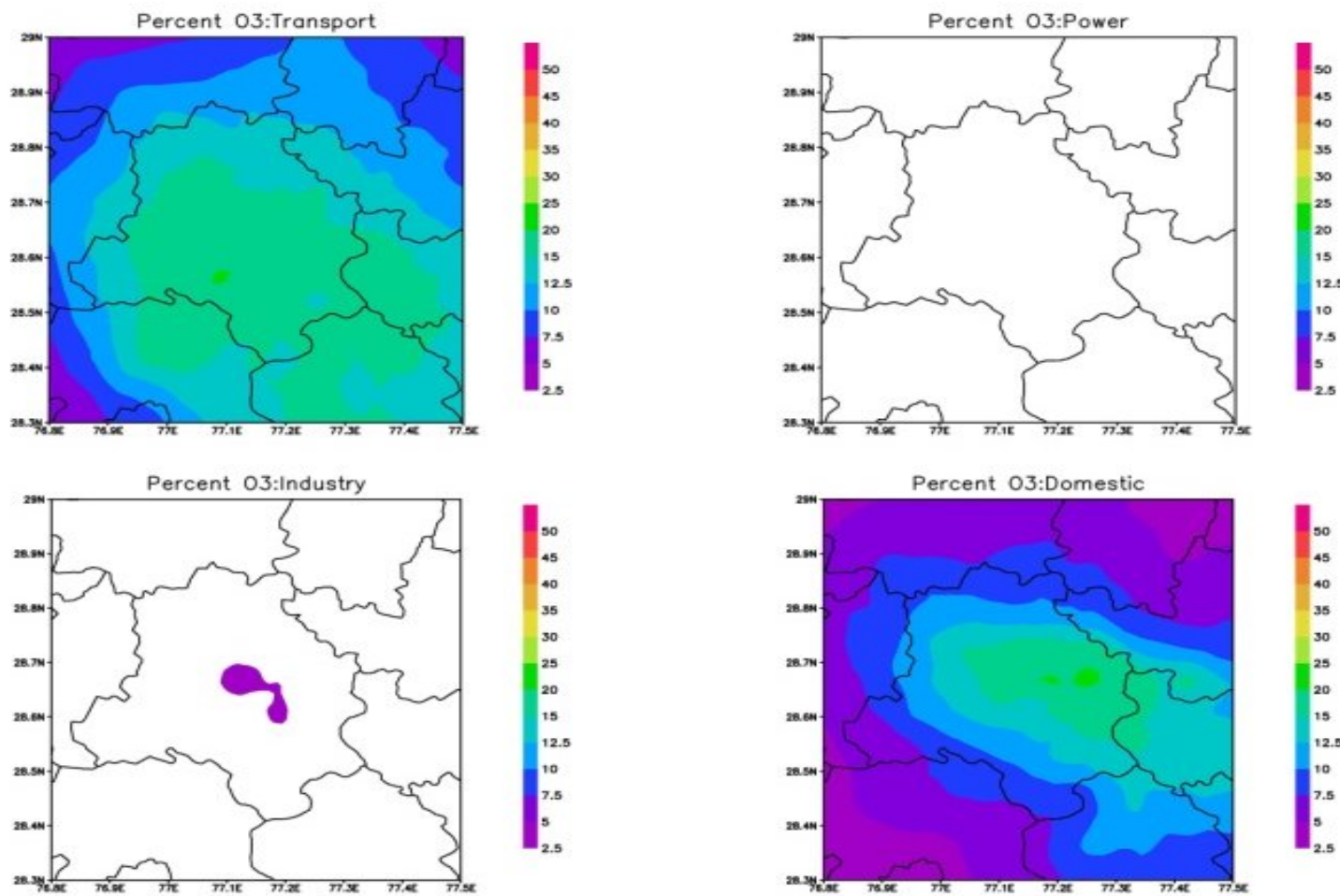

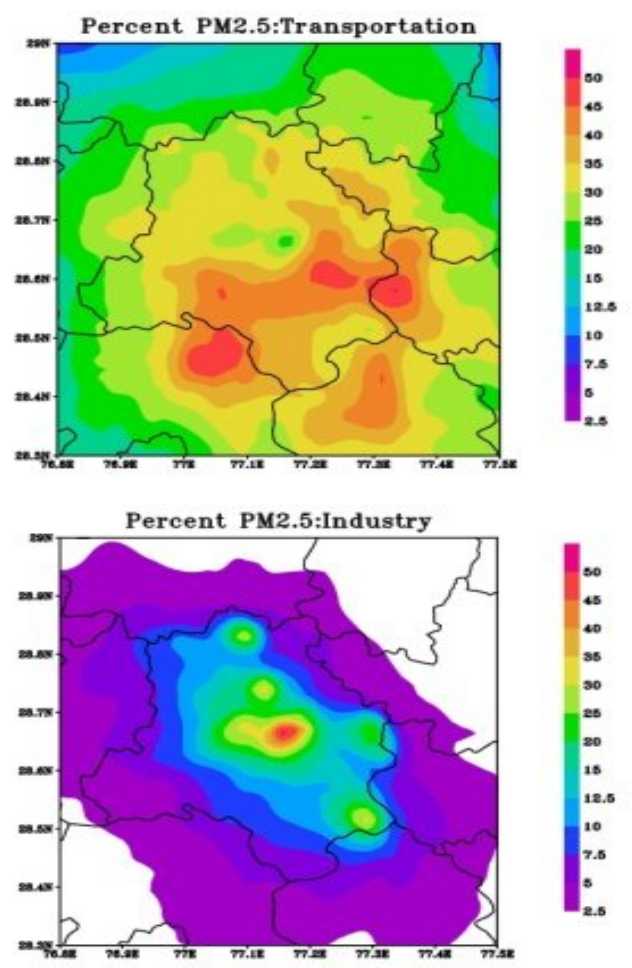

54
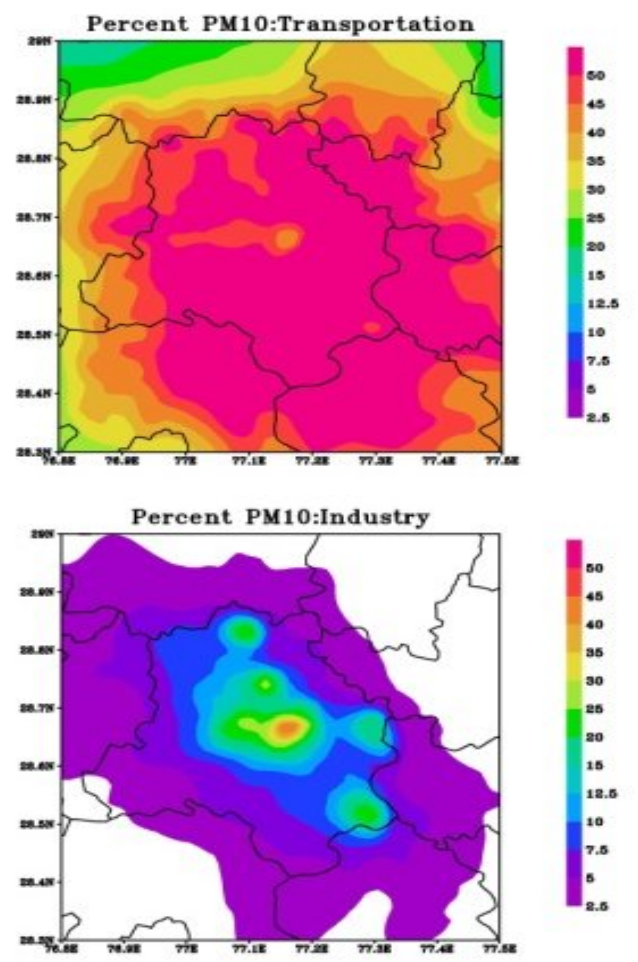
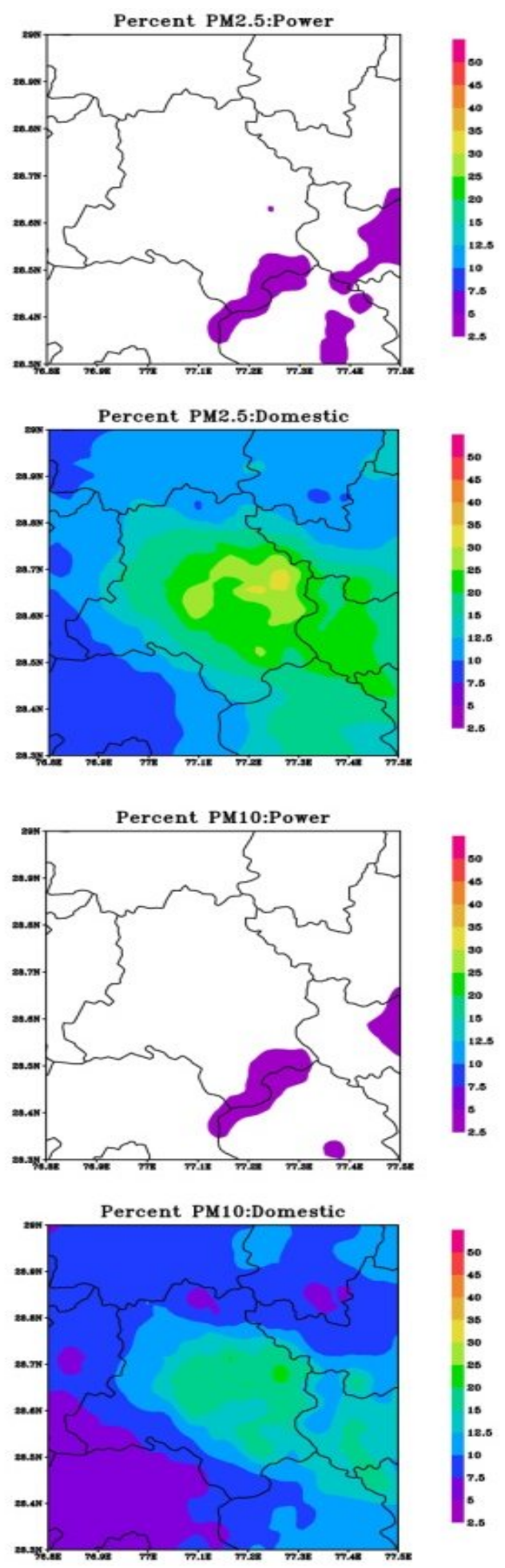

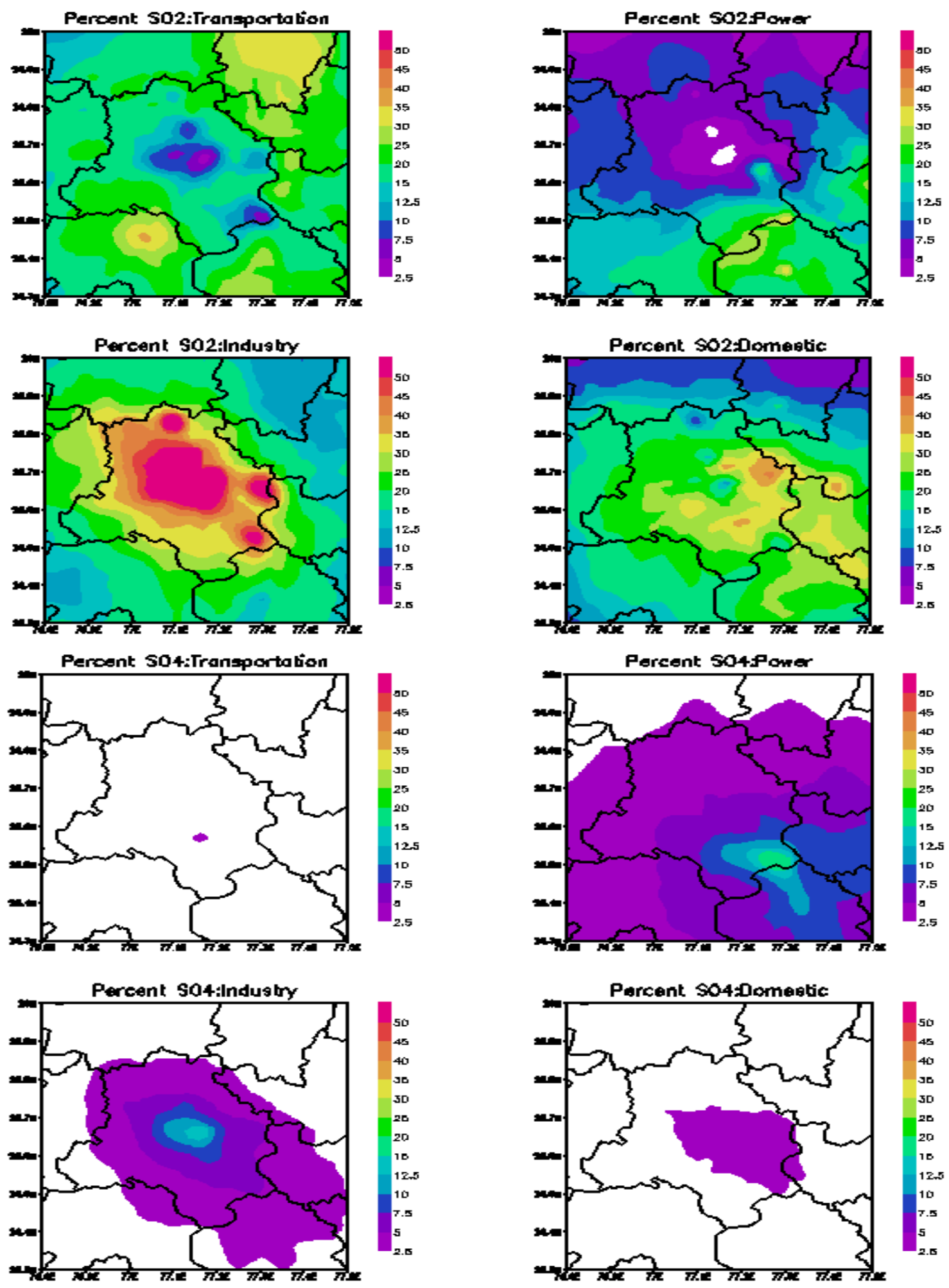

62 FigS2.Twenty day mean percent contribution of each pollutant from different sectors. 